\title{
Serum fatty acids and progression from dengue fever to dengue haemorrhagic fever/dengue shock syndrome
}

\author{
Eduardo Villamor ${ }^{1,2_{*}}$, Luis A. Villar ${ }^{3,4}$, Anyela Lozano-Parra ${ }^{3}$, Víctor M. Herrera ${ }^{3}$ and Oscar F. Herrán ${ }^{3}$ \\ ${ }^{1}$ Department of Epidemiology, University of Michigan School of Public Health, Ann Arbor, MI 48109, USA \\ ${ }^{2}$ Center for Human Growth and Development, University of Michigan, Ann Arbor, MI 48109, USA \\ ${ }^{3}$ Facultad de Salud, Centro de Investigaciones Epidemiológicas, Universidad Industrial de Santander, \\ Bucaramanga, Colombia \\ ${ }^{4}$ Departamento de Investigación y Desarrollo, Centro de Atención y Diagnóstico de Enfermedades Infecciosas (CDI), \\ Bucaramanga, Colombia
}

(Submitted 4 December 2017 - Final revision received 8 June 2018 - Accepted 17 June 2018 - First published online 14 August 2018)

\section{Abstract}

PUFA might modulate inflammatory responses involved in the development of severe dengue. We aimed to examine whether serum PUFA concentrations in patients diagnosed with dengue fever (DF) were related to the risk of progression to dengue haemorrhagic fever/dengue shock syndrome (DHF/DSS). A secondary aim was to assess correlations between fatty acids (FA) and inflammatory biomarkers in patients with DF. We conducted a prospective case-control study nested within a cohort of patients who were diagnosed with DF and followed during the acute episode. We compared the distribution of individual FA (\% of total FA) at onset of fever between 109 cases who progressed to DHF/ DSS and 235 DF non-progressing controls using unconditional logistic regression. We estimated correlations between baseline FA and cytokine concentrations and compared FA concentrations between the acute episode and >1 year post-convalescence in a subgroup. DHA was positively related to progression to DHF/DSS (multivariable adjusted OR (AOR) for DHA in quintile $5 v \cdot 1=5 \cdot 34,95 \%$ CI $2 \cdot 03,14 \cdot 1$; $P_{\text {trend }}=0 \cdot 007$ ). Dihomo- $\gamma$-linolenic acid (DGLA) was inversely associated with progression (AOR for quintile $5 v \cdot 1=0 \cdot 30,95 \%$ CI $0 \cdot 13,0 \cdot 69$; $\left.P_{\text {trend }}=0.007\right)$. Pentadecanoic acid concentrations were inversely related to DHF/DSS. Correlations of PUFA with cytokines at baseline were low. PUFA were lower during the acute episode than in a disease-free period. In conclusion, serum DHA in patients with DF predicts higher odds of progression to DHF/DSS whereas DGLA and pentadecanoic acid predict lower odds.

Key words: Fatty acids: PUFA: Dengue: Dengue haemorrhagic fever: Dengue shock syndrome

Dengue is the most common mosquito-borne viral infection worldwide, affecting as many as 100 million people annually ${ }^{(1,2)}$. The typical presentation of dengue is as a febrile illness with a variety of accompanying symptoms. In a few cases, the disease can progress to a life-threatening syndrome characterised by plasma leakage. The factors that induce progression from dengue fever (DF) to these potentially fatal forms of the disease, including dengue haemorrhagic fever (DHF) and dengue shock syndrome (DSS), are uncertain. They may involve secondary infections by new viral serotypes, agent virulence and host characteristics ${ }^{(3)}$

Among the host factors that could influence the risk of developing DHF/DSS, the nutritional status could play an important role because many nutrients serve key immunomodulatory functions. For example, vitamin E supplementation to patients with DF resulted in increased platelet counts in India ${ }^{(4)}$; 25-hydroxy vitamin D levels were positively related to severe dengue in Colombia ${ }^{(5)}$ and India ${ }^{(6)}$ whereas 1,25-hydroxyvitamin D was inversely associated with DHF/DSS in Nicaragua ${ }^{(7)}$, and $\mathrm{Zn}$ and $\mathrm{Cu}$ serostatus were related to dengue disease severity in cross-sectional studies in Indonesia ${ }^{(8)}$ and India ${ }^{(9)}$. Fatty acids (FA) are salient amidst the nutrients that could affect pathophysiological pathways leading to severe forms of dengue virus (DENV) infection. FA influence the organisation of cell membranes and the composition of lipid rafts through their incorporation as phospholipids and sphingolipids ${ }^{(10)}$. Lipid raft microdomains play an essential role in DENV protein synthesis and replication ${ }^{(11)}$. In addition, some $n-3$ PUFA, including EPA (20:5n-3) and DHA (22:6n-3) could reduce expression of pro-inflammatory cytokines through different mechanisms including down-regulated gene expression in mononuclear cells, decreased synthesis of pro-inflammatory

Abbreviations: AA, arachidonic acid; D5D, $\Delta 5$-desaturase; DENV, dengue virus; DF, dengue fever; DGLA, dihomo- $\gamma$-linolenic acid; DHF, dengue haemorrhagic fever; DSS, dengue shock syndrome; FA, fatty acid; SCD, stearoyl-coA-desaturase.

* Corresponding author: E. Villamor, email villamor@umich.edu 
eicosanoids derived from $n$-6 PUFA, reduced chemotaxis and lymphocyte proliferation, enhanced apoptosis of Th- 1 cells, and decreased endothelial activation and dysfunction ${ }^{(12)}$. Some $n-6$ PUFA, including arachidonic acid (AA, 20:4n-6), are considered pro-inflammatory while others such as dihomo- $\gamma$-linolenic acid (DGLA, 20:3n-6) exhibit anti-inflammatory properties $^{(13)}$. Despite the potential of FA to regulate pathways involved in the aetiology of severe dengue, they have not been systematically studied in the context of this infection. Some investigations indicate that FA concentrations are altered during the acute stages of dengue infection ${ }^{(14,15)}$. Because therapies to prevent progression of DF to DHF/DSS are virtually inexistent, identifying modifiable factors associated with risk of these severe forms of the disease is a high research priority.

We conducted a prospective case-control study nested in a cohort of patients who were diagnosed with DF and followed during the acute episode. The primary aim was to investigate the associations of FA concentrations in serum collected $<96 \mathrm{~h}$ from the onset of fever with progression to DHF/DSS. A secondary aim was to determine whether serum FA could be affected by acute inflammation at the early stages of DF. This was realised by assessing the correlations between inflammatory biomarkers and FA and by comparing FA concentrations during the acute episode and a disease-free period, after $>1$ year post-convalescence from the disease.

\section{Methods}

\section{Study design}

This was a case-control study nested within a cohort of patients diagnosed with DF who were followed during the acute episode. Ambulatory patients with suspected dengue were recruited during non-epidemic (May 2003-September 2009) and epidemic (October 2009-December 2010) periods at healthcare centres in five areas of Bucaramanga, a city in northeast Colombia. Eligible participants had an acute febrile syndrome caused by DENV infection, had onset of symptoms $<96$ h before consultation, and were $\geq 5$ years of age. Exclusion criteria were as follows: history of diabetes, acquired immunodeficiency syndrome, haematological disorders, cancer or CVD; or, at baseline, DHF or DSS (case definition below), major bleeding, hypoalbuminaemia, effusions or shock. At the time of recruitment, we elicited information on sociodemographic characteristics, medical history and symptoms through a questionnaire. Height and weight were measured on calibrated instruments with the use of standardised techniques and a complete physical examination was carried out. Blood samples were obtained to determine haematocrit, platelet counts and albumin concentration. To identify the occurrence of DHF/DSS, we followed participants during the acute episode daily at their homes until the 7th day of disease or the day of hospital discharge if they were admitted. Data collected during this period included signs and symptoms of DHF/DSS, as well as daily haematocrit measures. To detect thrombocytopenia, a DHF/ DSS diagnostic criterion, we started daily platelet count measurements when patients had spontaneous haemorrhage, signs of effusion, oedema, a haematocrit change $>10 \%$ or a platelet count $<120000 / \mathrm{mm}^{3}$. We saw the patients again in the convalescent period, 7-15d after the onset of symptoms, and obtained a new blood sample. DENV infection was confirmed according to a diagnostic algorithm that included IgM seroconversion from the acute to the convalescent samples (shift from negative to positive or a titre increase $\geq 4$ ) plus either a positive result for NS1 antigen or viral genome amplification per RT-PCR in acute serum $(<96 \mathrm{~h}$ from the onset of fever). IgM antibodies were determined with use of an enzyme-linked immunosorbent assay (Panbio Dengue IgM Capture ELISA; Alere), NS1 antigen was quantified with the ELISA NS1 Dengue kit (Panbio; Alere), and RT-PCR amplification of viral RNA was conducted with the QIAamp Viral RNA kit (Qiagen). We determined whether DENV infection was secondary with use of the Panbio Dengue IgG Capture ELISA test (Alere).

Case definition. Cases were patients who developed DHF or DSS during follow-up, according to the 1997 WHO criteria, prevalent at the time ${ }^{(16)}$. DHF cases met all of the following criteria: a platelet count $\leq 100000 / \mathrm{mm}^{3}$, any spontaneous haemorrhage or $\geq 1$ positive tourniquet test, and evidence of plasma leakage (i.e. pleural effusion, ascites, hypoalbuminaemia $<30 \mathrm{~g} / \mathrm{l}$ or an increase in haematocrit $>10 \%$ ). A haemoconcentration of $10 \%$, instead of $20 \%$, was chosen because this criterion has greater sensitivity in identifying dengue-related complications ${ }^{(17)}$ and has been associated with severe morbidity in patients from areas endemic for dengue ${ }^{(18)}$. DSS cases had all criteria above plus any reading of mean arterial pressure $<70 \mathrm{mmHg}$ or pulse pressure $<20 \mathrm{mmHg}$ during follow-up.

The study was conducted according to the guidelines laid down in the Declaration of Helsinki. All participants gave written informed consent before recruitment. Among children, written consent was sought from the primary care provider and assent from the children was confirmed before recruitment. The study protocol was approved by the Medical Ethics Committee of the Industrial University of Santander. The University of Michigan Health Sciences and Behavioral Sciences Institutional Review Board approved the use of data and samples from the study.

\section{Study population}

We recruited 820 participants into the cohort. Of them, 173 (21.1\%) developed DHF/DSS during follow-up. For the casecontrol study, we selected all 109 cases $(63.0 \%$ of all cases in the cohort) whose first available serum sample (at the time of the initial consultation) had been collected within $96 \mathrm{~h}$ from the onset of fever ('acute' serum sample) and had sufficient volume for FA quantification. Next, we randomly selected a group of controls among the patients who did not reach DHF/DSS criteria during follow-up, using a 2:1 ratio of controls per case plus an additional $10 \%$ in anticipation of losses due to lack of an acute serum sample. The final number of controls was 235 . Selected controls were comparable to those not selected with respect to sex, age, socio-economic status, hours with fever before consultation and signs of severity. Nevertheless, selected controls were more likely to have become infected during the 
epidemic period and were taller and heavier compared with non-selected controls. Selected cases were compared with nonselected cases in the same manner as controls.

A post-convalescence blood sample was obtained in a subgroup of fifteen cases and twenty-nine controls who were visited at home after a median 1.8 years (range $1.3-2.5$ ) from the acute episode.

\section{Laboratory methods}

Serum fatty acids. Samples were shipped frozen to the University of Michigan where total serum FA were quantified at the Regional Comprehensive Metabolomics Resource Core. Total lipids were extracted from $200 \mu \mathrm{l}$ serum per the methods described by Bligh \& Dyer ${ }^{(19)}$. About $10 \mu \mathrm{l}$ of $4 \mathrm{~mm}$ nonadecanoic acid (C19:0) was added as an internal standard. Boron trifluoride-methanol was used to derivatise the FA portion of the total lipids into their methyl esters as previously described $^{(20)}$. To extract the methyl esters, a 2:1 hexane-water mixture was added and the sample was centrifuged. The hexane layer containing the methyl esters was removed from the aqueous layer and dried, and the methyl esters were resuspended in 100-200 $\mu \mathrm{l}$ of hexane, according to the volume of the original sample. About 1-2 $\mu \mathrm{l}$ was injected via an autosampler and analysed on a gas chromatographer (Model 6890N; Agilent) equipped with a flame ionisation detector and a $100 \mathrm{~m} \times$ $0.25 \mathrm{~mm} \times 0.2 \mu \mathrm{m}$ SP-2560 column (Sigma-Aldrich). To quantify FA, known amounts of C19:0 and other authentic methyl esters were used to create a calibration curve. The authentic methyl esters were also used to identify FA in samples based on their retention times. Eluted peaks were analysed with Chemstation software (Agilent). FA concentrations were expressed as the percentage of each FA relative to the total FA concentration (FA \%). This FA \% was estimated by dividing the area of each FA by the total area. The limit of detection for pentadecanoic acid (C15: 0), a type of FA present at low concentrations, was 50 pmol.

The activity of key enzymes in FA metabolism was estimated from desaturation and elongation indices calculated as the product/substrate ratios: stearoyl-coA-desaturase (SCD) as $18: 1 n-9 / 18: 0$, elongase as $18: 1 n-7 / 16: 1 n-7, \Delta 6$-desaturase as $18: 3 n-6 / 18: 2 n-6$ and $\Delta 5$-desaturase (D5D) as $20: 4 n-6 / 20: 3 n-6$.

Cytokines. We determined concentrations of pro- and antiinflammatory cytokines that may be independent predictors of progression to DHF/DSS. Interferon (IFN)- $\gamma$, IL-10, IL-6 and TNF- $\alpha$ were measured at the University of Michigan Cancer Center Immunology Core with the use of Luminex assays (Thermo Fisher Scientific Inc.) in all acute sera and in the samples collected post-convalescence.

Dengue virus serotype. DENV serotypes were identified in a subsample of forty-nine controls and twenty-eight cases using conventional and real time RT-PCR assays. Viral RNA was isolated from serum using the commercial kit QIAamp ${ }^{\circledR}$ Viral RNA. Conventional RT-PCR tests were performed following the Lanciotti protocol ${ }^{(21)}$. Real-time RT-PCR were conducted with the CDC Kit DENV-1-4 Real-Time RT-PCR (Centers for Disease Control and Prevention) ${ }^{(22)}$.

\section{Data analysis}

Variables. Case (DHF/DSS) status was the outcome of interest. FA \% were the primary exposures and enzyme activity indices were secondary exposures. We considered as covariates baseline characteristics that could confound the associations between FA status and progression to DHF/DSS. These included sociodemographic (age, sex, socio-economic status), anthropometric (height and BMI as nutritional status indicators), clinical (time since the beginning of fever, early signs of severity), virological (secondary infection, serotype) and immunological (cytokine concentrations) characteristics.

Comparison of controls and cases. We first compared the distribution of baseline covariates between controls and DHF/ DSS cases, which were categorised as presented in Table 1. Case-control differences were tested with the use of $\chi^{2}$ and Wilcoxon rank-sum tests for categorical and continuous characteristics, respectively.

Fatty acids and progression to dengue haemorrhagic fever/ dengue shock syndrome. We compared the distribution of FA between controls and cases using means and standard deviations and the Wilcoxon rank-sum test. Next, we categorised each FA into quintiles according to the distribution among controls and estimated OR with 95\% CI with the use of unconditional logistic regression. Tests for linear trend were conducted by introducing into the models a variable representing the median values of each quintile as a continuous predictor. Multivariable-adjusted OR and 95\% CI were estimated for each FA in models that included as covariates sex, age, hours with fever before consultation, and IL-10 and TNF- $\alpha$ concentrations at the time of consultation. Finally, we fitted a model with simultaneous adjustment for the FA that were statistically significant predictors of DHF/DSS progression in multivariable analyses and that remained significant in the final model at $P<0.05$.

Acute inflammation and fatty acids. To ascertain whether acute inflammation in the course of DENV infection was related to FA concentrations, we estimated sex- and age-adjusted partial Spearman's correlation coefficients between the cytokines (IFN- $\gamma$, IL-10, IL-6 and TNF- $\alpha$ ) and each FA separately for controls and cases. Next, we estimated the correlations between the analytes measured in the samples collected after $>1$ year post-convalescence. We also examined the prevalence of thrombocytopenia at baseline by FA quintiles since platelet count is a strong predictor of progression to severe dengue.

Change in fatty acid concentrations from the acute episode to post-convalescence. In the subgroup with a postconvalescence blood sample, we estimated the difference in FA concentrations between after $>1$ year of recovery and the acute 
Table 1. Characteristics of dengue fever controls and dengue haemorrhagic fever/dengue shock syndrome cases at the time of uncomplicated dengue fever diagnosis (baseline)

(Mean values and standard deviations; medians and ranges)

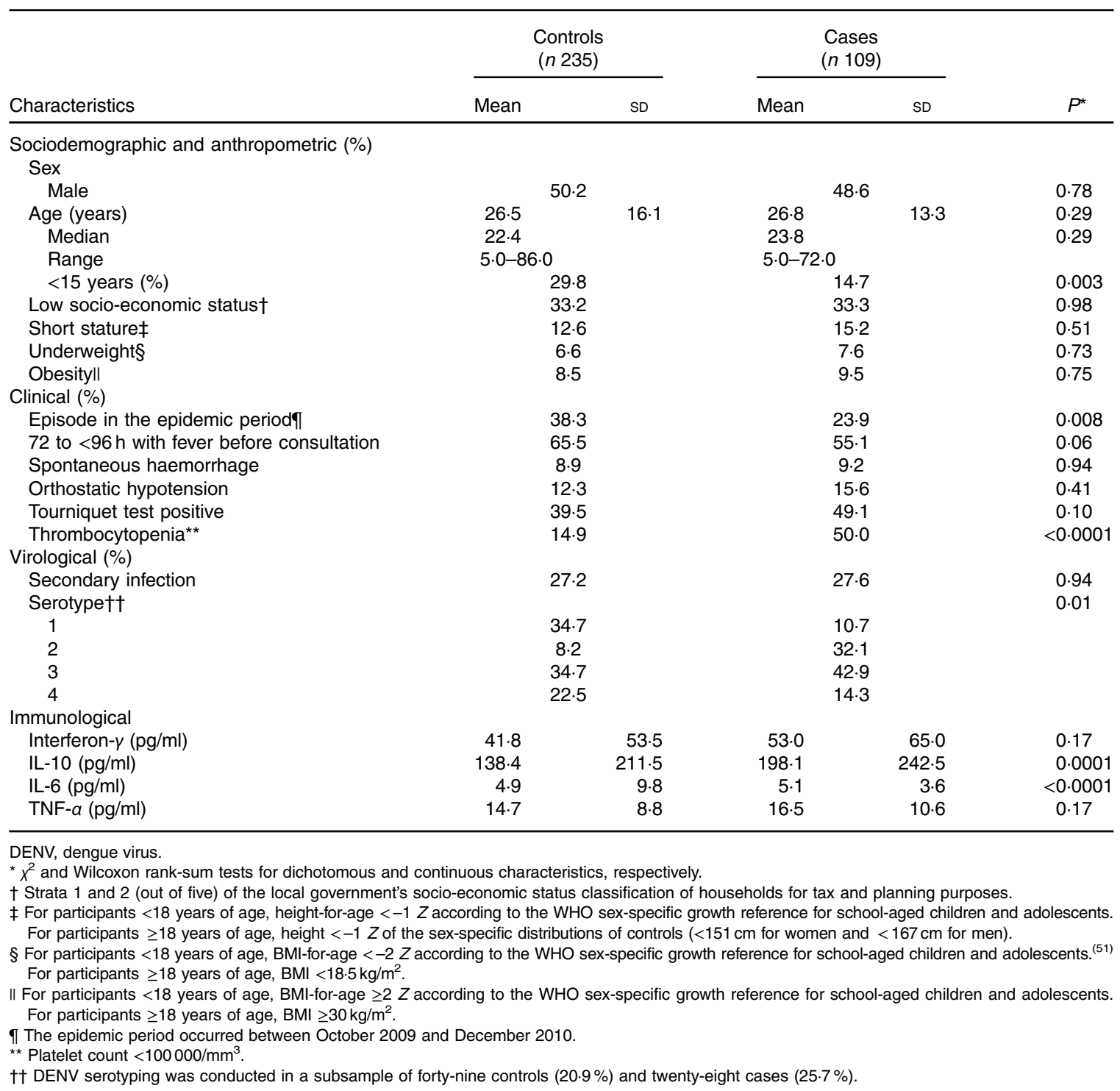

episode with the use of a repeated-measures linear regression model with each FA as the continuous outcome and order of measurement as the predictor. An exchangeable covariance matrix was specified to account for within-subject correlations. Participants with a post-convalescence sample were more likely to have been recruited during the epidemic period, were younger and of lower socio-economic status, and consulted later after the onset of fever than those without these samples. They also differed with respect to some of the FA at baseline.

Associations of dengue virus serotype with fatty acids. Because DENV serotype is a strong independent predictor of progression to severe dengue ${ }^{(23)}$ and we lacked this information on the majority of participants, we examined the associations of serotype with serum FA in the subset with available data to explore whether serotype could have confounded the key associations between FA and case status. We compared the distribution of FA by serotype with the use of Wilcoxon ranksum tests.

All analyses were carried out with Statistical Analysis Software version 9.4 (SAS Institute Inc.).

\section{Results}

\section{Characteristics of controls and cases}

In all, $50 \%$ ( $n$ 173) of participants were female. Age was $26 \cdot 6$ (sD $15 \cdot 2$ ) years (range $5-86$ ); $25 \%$ of participants ( $n$ 86) were $<15$ years old. Compared with controls, cases were older, consulted earlier after the onset of fever, had lower platelet counts and had higher cytokine concentrations at baseline (Table 1). 
Fatty acids and progression to dengue haemorrhagic fever/ dengue shock syndrome

Mean pentadecanoic (15:0) and stearic (18:0) acid concentrations were significantly lower in cases compared with controls (Table 2, online Supplementary Fig. S1), whereas behenic acid (22:0) levels were higher. There were no significant differences in MUFA concentrations by case status. Mean concentrations of the long-chain $n$-3 PUFA docosapentaenoic acid (DPA) and DHA and of the $n$ - 6 PUFA DGLA, AA and adrenic acid were significantly higher in cases compared with controls (Table 2, online Supplementary Fig. S1). SCD and D5D activities were higher in cases than controls. When the FA exposures were considered in quintiles of their distributions among controls (online Supplementary Table S1), pentadecanoic acid and DGLA were inversely associated with case status whereas DHA, AA, SCD and D5D were positively related to progression to DHF/DSS, after adjustment for sex, age, hours with fever before consultation and IL-10 and TNF- $\alpha$ concentrations. In a multivariable model adjusted for these FA, simultaneously pentadecanoic acid, DHA and DGLA remained significantly associated with progression to DHF/DSS (Table 3) whereas the associations with AA and SCD were attenuated and became non-statistically significant. For pentadecanoic acid, compared with patients in the lowest quintile those in the highest

Table 2. Distribution of serum fatty acids (FA) percentage in dengue fever controls and dengue haemorrhagic fever/dengue shock syndrome cases

(Mean values and standard deviations)

\begin{tabular}{|c|c|c|c|c|c|}
\hline \multirow[b]{2}{*}{ FA } & \multicolumn{2}{|c|}{$\begin{array}{l}\text { Controls } \\
(n \text { 235) }\end{array}$} & \multicolumn{2}{|c|}{$\begin{array}{c}\text { Cases } \\
(n 109)\end{array}$} & \multirow[b]{2}{*}{$P^{\star}$} \\
\hline & Mean & SD & Mean & SD & \\
\hline \multicolumn{6}{|l|}{ SFA (\%) } \\
\hline 14:0 myristic acid & 0.35 & 0.22 & 0.30 & 0.16 & 0.12 \\
\hline $15: 0$ pentadecanoic acid & 0.16 & 0.06 & 0.15 & 0.06 & 0.02 \\
\hline $16: 0$ palmitic acid & 23.43 & $2 \cdot 31$ & $23 \cdot 15$ & 2.06 & 0.17 \\
\hline $17: 0$ margaric acid & 0.37 & 0.08 & 0.36 & 0.11 & 0.08 \\
\hline $18: 0$ stearic acid & 8.14 & $1 \cdot 10$ & $7 \cdot 70$ & 1.04 & 0.005 \\
\hline $20: 0$ arachidic acid & 0.16 & 0.06 & 0.16 & 0.04 & 0.24 \\
\hline 22: 0 behenic acid & 0.33 & 0.11 & 0.36 & 0.13 & 0.02 \\
\hline 24:0 lignoceric acid & 0.28 & 0.14 & 0.30 & 0.14 & 0.11 \\
\hline Total SFA & $33 \cdot 21$ & $2 \cdot 80$ & 32.47 & 2.48 & 0.02 \\
\hline \multicolumn{6}{|l|}{ MUFA (\%) } \\
\hline $16: 1 n-7$ palmitoleic acid & 1.82 & 0.61 & 1.77 & 0.54 & 0.79 \\
\hline $18: 1 n-9$ oleic acid & $23 \cdot 30$ & $2 \cdot 81$ & $23 \cdot 62$ & 2.98 & 0.54 \\
\hline 18:1n-7 cis-vaccenic acid & $2 \cdot 28$ & 0.56 & 2.36 & 0.59 & 0.08 \\
\hline $20: 1 n-9$ gondoic acid & 0.19 & 0.07 & 0.20 & 0.06 & 0.30 \\
\hline $24: 1 n-9$ nervonic acid & 0.43 & 0.25 & 0.45 & 0.23 & 0.14 \\
\hline Total MUFA & $28 \cdot 13$ & $3 \cdot 20$ & 28.48 & 3.31 & 0.63 \\
\hline \multicolumn{6}{|l|}{ n-3 PUFA (\%) } \\
\hline $18: 3 n-3 a$-linolenic acid & 0.29 & 0.18 & 0.25 & 0.13 & 0.09 \\
\hline $20: 5 n-3$ EPA & 0.27 & 0.12 & 0.30 & 0.14 & 0.22 \\
\hline $22: 5 n-3$ DPA & 0.39 & 0.15 & 0.43 & 0.17 & 0.03 \\
\hline $22: 6 n-3 \mathrm{DHA}$ & 1.50 & 0.72 & 1.72 & $1 \cdot 11$ & 0.01 \\
\hline Total long-chain $n-3$ PUFA† & $2 \cdot 16$ & 0.80 & 2.45 & $1 \cdot 15$ & 0.005 \\
\hline Total $n-3$ PUFA & 2.53 & 0.92 & 2.76 & $1 \cdot 18$ & 0.05 \\
\hline \multicolumn{6}{|l|}{$n-6$ PUFA (\%) } \\
\hline $18: 2 n-6$ linoleic acid & $26 \cdot 42$ & 3.82 & 25.97 & 3.34 & 0.45 \\
\hline $18: 3 n-6 \gamma$-linolenic acid & 0.24 & 0.14 & 0.26 & 0.13 & 0.19 \\
\hline $20: 2 n-6$ EDA & 0.23 & 0.12 & 0.24 & 0.09 & 0.41 \\
\hline $20: 3 n-6$ dihomo- $\gamma$-linolenic acid & 1.38 & 0.42 & 1.30 & 0.44 & 0.03 \\
\hline $20: 4 n-6$ arachidonic acid & 6.08 & 1.47 & 6.62 & 1.48 & 0.006 \\
\hline $22: 4 n-6$ adrenic acid & 0.48 & 0.39 & 0.73 & 0.97 & 0.006 \\
\hline Total long-chain $n$-6 PUFA $\ddagger$ & 8.17 & 1.70 & 8.89 & $2 \cdot 01$ & 0.006 \\
\hline Total $n-6$ PUFA & 34.83 & 4.51 & $35 \cdot 12$ & $4 \cdot 11$ & 0.39 \\
\hline $18: 2 n-7 c t$ CLA (\%) & 0.06 & 0.11 & 0.05 & 0.10 & 0.13 \\
\hline \multicolumn{6}{|l|}{ trans-FA (\%) } \\
\hline $16: 1 n-7$ trans & 0.23 & 0.15 & 0.22 & 0.09 & 0.92 \\
\hline $18: 1$ trans & 0.62 & 0.50 & 0.56 & 0.46 & 0.12 \\
\hline $18: 2$ trans & 0.38 & 0.25 & 0.35 & 0.17 & 0.28 \\
\hline Total trans-FA & $1 \cdot 23$ & 0.75 & $1 \cdot 13$ & 0.60 & 0.14 \\
\hline \multicolumn{6}{|l|}{ Enzyme activity indices } \\
\hline Stearoyl-coA-desaturase $18: 1 n-9 / 18: 0$ & 2.91 & 0.51 & 3.14 & 0.68 & 0.008 \\
\hline Elongase $18: 1 n-7 / 16: 1 n-7$ & 1.39 & 0.65 & 1.48 & 0.71 & 0.11 \\
\hline$\Delta 6$-Desaturase $18: 3 n-6 / 18: 2 n-6$ & 0.009 & 0.006 & 0.100 & 0.005 & 0.20 \\
\hline$\Delta 5$-Desaturase $20: 4 n-6 / 20: 3 n-6$ & 4.79 & 1.98 & 5.58 & $2 \cdot 17$ & 0.0001 \\
\hline
\end{tabular}

DPA, docosapentaenoic acid; EDA, eicosadienoic acid; CLA, conjugated linoleic acid.

* Wilcoxon rank-sum test.

† Sum of 20:5n-3 EPA, 22:5n-3 DPA and $22: 6 n-3$ DHA.

¥ Sum of $20: 2 n-6$ EDA, $20: 3 n-6$ dihomo- $\gamma$-linolenic acid, $20: 4 n-6$ arachidonic acid and $22: 4 n-6$ adrenic acid. 
Table 3. Adjusted OR for progression to dengue haemorrhagic fever/dengue shock syndrome according to baseline characteristics and serum fatty acids (FA) percentage

(Odds ratios and $95 \%$ confidence intervals)

\begin{tabular}{|c|c|c|c|c|c|c|c|c|}
\hline Characteristic & Controls & Cases & Unadjusted & $\mathrm{OR}^{*}$ & $95 \% \mathrm{Cl}$ & Adjusted OR† & $95 \% \mathrm{Cl}$ & Adjusted $P \ddagger$ \\
\hline Sex & & & & & & & & 0.85 \\
\hline Female & 117 & 56 & & 1.00 & & 1.0 & & \\
\hline Male & 118 & 53 & 0.94 & & $0.60,1.48$ & 0.95 & $0.57,1.58$ & \\
\hline Age (years) & & & & & & & & \\
\hline$<15$ & 165 & 93 & & 1.00 & & 1.0 & & 0.003 \\
\hline$\geq 15$ & 70 & 16 & 2.47 & & $1.35,4.49$ & $2 \cdot 75$ & $1.41,5.34$ & \\
\hline Hours with fever before consultation & & & & & & & & 0.02 \\
\hline$<72$ & 81 & 49 & & 1.00 & & 1.0 & & \\
\hline 72 to $<96$ & 154 & 60 & 0.64 & & $0.41,1.02$ & 0.52 & $0.31,0.89$ & \\
\hline IL-10, per $200 \mathrm{pg} / \mathrm{ml}$ & _ & & 1.25 & & $1.03,1.53$ & 1.23 & $0.97,1.57$ & 0.09 \\
\hline TNF- $a$, per $10 \mathrm{pg} / \mathrm{ml}$ & - & & 1.22 & & $0.97,1.55$ & 1.31 & $0.98,1.74$ & 0.07 \\
\hline $15: 0$ pentadecanoic acid quintile§, $\%$ (median) & & & & & & & & 0.002 \\
\hline Q1 (0.11) & 46 & 27 & & 1.00 & & 1.0 & & \\
\hline Q2 (0.14) & 47 & 27 & 0.98 & & $0.50,1.91$ & 0.67 & $0.32,1.40$ & \\
\hline Q3 $(0.16)$ & 47 & 23 & 0.83 & & $0.42,1.66$ & 0.55 & $0.26,1.19$ & \\
\hline Q4 (0.19) & 47 & 22 & 0.80 & & $0.40,1.60$ & 0.53 & $0.24,1.15$ & \\
\hline Q5 (0.24) & 47 & 9 & 0.33 & & $0.14,0.77$ & 0.24 & $0.09,0.60$ & \\
\hline 22 : 6n-3 DHA quintile§, \% (median) & & & & & & & & 0.007 \\
\hline Q1 $(0.81)$ & 47 & 7 & & 1.00 & & 1.0 & & \\
\hline Q2 (1.11) & 47 & 26 & $3 \cdot 71$ & & $1.47,9.39$ & 4.44 & $1.67,11.8$ & \\
\hline Q3 (1.37) & 47 & 20 & 2.86 & & $1 \cdot 10,7 \cdot 39$ & 4.57 & $1 \cdot 64,12 \cdot 7$ & \\
\hline Q4 (1.68) & 47 & 24 & 3.43 & & $1.35,8.72$ & $5 \cdot 58$ & $2.01,15 \cdot 4$ & \\
\hline Q5 (2.26) & 47 & 32 & 4.57 & & $1.84,11.4$ & $5 \cdot 34$ & $2 \cdot 03,14 \cdot 1$ & \\
\hline $20: 3 n-6$ dihomo- $\gamma$-linolenic acid quintile§, \% (median) & & & & & & & & 0.007 \\
\hline Q1 $(0.91)$ & 47 & 31 & & 1.00 & & 1.0 & & \\
\hline Q2 (1.14) & 47 & 22 & 0.71 & & $0.36,1.40$ & 0.51 & $0.24,1.09$ & \\
\hline Q3 (1.34) & 47 & 23 & 0.74 & & $0.38,1.46$ & 0.58 & $0.27,1.24$ & \\
\hline Q4 (1.55) & 47 & 17 & 0.55 & & $0.27,1.12$ & 0.43 & $0.19,0.96$ & \\
\hline Q5 (1.98) & 47 & 16 & 0.52 & & $0.25,1.07$ & 0.30 & $0.13,0.69$ & \\
\hline$\Delta 5$-Desaturasell quintile (median) & & & & & & & & 0.0004 \\
\hline Q1 (2.89) & 47 & 15 & & 1.00 & & 1.0 & & \\
\hline Q2 (3.80) & 47 & 12 & 0.80 & & $0.34,1.89$ & 0.97 & $0.38,2.50$ & \\
\hline Q3 (4.50) & 47 & 14 & 0.93 & & $0.41,2 \cdot 15$ & 0.85 & $0.34,2 \cdot 12$ & \\
\hline Q4 (5.16) & 47 & 22 & 1.47 & & $0.68,3.17$ & 1.40 & $0.60,3.28$ & \\
\hline Q5 (6.77) & 47 & 46 & 3.07 & & $1.51,6.23$ & 3.28 & $1.42,7.58$ & \\
\hline
\end{tabular}

${ }^{*}$ From unconditional logistic regression models with case status (dengue haemorrhagic fever/dengue shock syndrome) as the outcome and each covariate presented as predictor. † From an unconditional logistic regression model with case status as the outcome and predictors that included sex (one indicator for male), age (one indicator for $<15$ years), hours with fever before consultation (one indicator for $\geq 72 \mathrm{~h}$ ), IL-10 (continuous), TNF-a (continuous), 15:0 pentadecanoic acid quintile (four indicators), $22: 6 n-3 \mathrm{DHA}$ quintile (four indicators) and $20: 3 n-6$ dihomo- $\gamma$-linolenic acid quintile (four indicators). Estimates for $\Delta 5$-desaturase were obtained from a model that excluded $20: 3 n$ - 6 dihomo- $\gamma$-linolenic acid due to collinearity.

‡ For sex, age, hours with fever before consultation and cytokines, Wald test. For FA, test for linear trend when a variable representing the median of each quartile was introduced into the logistic regression model as a continuous predictor.

$\S$ Percentage of total serum FA.

II Ratio of $20: 4 n-6 / 20: 3 n-6$

had a $76 \%$ lower odds of progression $(P=0 \cdot 002)$. DHA was positively related to case status in a non-linear manner; compared with patients in the lowest quintile, odds of progression were five times higher (OR: 4.96; $95 \%$ CI 2.05, 11.99; $P=0.0004$ ) for patients with concentrations in the second quintile or above. DGLA was inversely related to DHF/DSS progression; the OR (95\% CI) between quintiles 5 and 1 was 0.30 (95\% CI 0.13, 0.69; $P=0.005)$. When D5D was substituted for DGLA in the model, D5D was positively associated with DHF/DSS progression (Q5 $v$. Q1 OR: 3.28; 95\% CI 1.42, 7.58; $P=0 \cdot 006$ ).

\section{Acute inflammation and fatty acids}

Correlations between FA and cytokine concentrations were generally low (online Supplementary Table S2). DGLA was weakly, inversely correlated with IL-10 at baseline but not postconvalescence. DPA, DHA and D5D were related to low platelet counts, whereas the $n$ - 6 PUFA $\gamma$-linolenic acid (GLA, $18: 3 n-6)$ and DGLA were associated with less thrombocytopenia (online Supplementary Table S3). Pentadecanoic acid was not related to platelet counts at baseline.

\section{Change in fatty acid concentrations from the acute episode to post-convalescence}

Among the forty-four patients with post-convalescent samples, concentrations of most SFA, MUFA and $n-3$ and n-6 PUFA changed from the time of the acute episode (online Supplementary Table S4). Concentrations of SFA, except for palmitic acid, tended to increase from the acute episode to postconvalescence, whereas MUFA concentrations, except for palmitoleic acid, decreased. $\alpha$-Linolenic acid (ALA), EPA, DPA and all $n-6$ PUFA concentrations increased. DHA was higher during the acute episode than post-convalescence, but the change was 
not statistically significant. There were no changes in trans FA. D5D activity decreased. Changes did not differ by case status during the acute episode (online Supplementary Table S5).

\section{Dengue virus serotype and fatty acids}

The distributions of some FA varied significantly by DENV serotype in the subset with available data (online Supplementary Table S6). Of the FA significantly associated with progression to DHF/DSS, only DHA was related to DENV serotype. DHA of participants infected with serotypes 2 or 3 was lower than that of patients infected with DENV 1 or 4 .

\section{Discussion}

In this prospective investigation of patients diagnosed with DF, serum DHA at the time of diagnosis was related to increased odds of progression to DHF/DSS whereas the SFA pentadecanoic acid and the n-6 PUFA DGLA were associated with decreased odds. In addition, increased D5D activity was related to higher odds of progression.

The positive association of DHA levels with progression to DHF/DSS is consistent with results from a small untargeted metabolomics study ${ }^{(7)}$. Serum DHA within $4 \mathrm{~d}$ from the onset of fever was higher in sixteen Nicaraguan children diagnosed with DF who progressed to DHF/DSS compared with fifteen children who did not progress. This finding is unexpected because DHA contributes to the resolution of inflammation through different pathways $^{(12)}$ and effective resolution of inflammation should be related to less risk of progression. Nevertheless, the association should also be interpreted in light of the comparison of DHA levels during the acute episode and post-convalescence. In our study, DHA was higher during the acute episode than in a presumably disease-free period. Although this difference was not statistically significant, it is in line with findings from a metabolomics study of adults with DF in which DHA was higher during the febrile stage of infection than it was 3-4 weeks after resolution of the episode ${ }^{(14)}$. An increase in DHA levels during acute DF has been interpreted as an early attempt to initiate the resolution of inflammation, which could start concomitantly with the initial proinflammatory response ${ }^{(7,14)}$. A more severe inflammatory process during the early stages of DF predicts progression to DHF/DSS; thus, a correspondingly more aggressive anti-inflammatory response involving increased DHA levels could explain the association of DHA with progression even in the absence of an adverse causal effect of DHA. Of note, however, in our data, DHA was generally not correlated with cytokine concentrations and the association of DHA with progression was observed even after controlling for these inflammation biomarkers. An adverse effect of DHA cannot be discarded. Rodent models indicate that $n-3$ long-chain PUFA supplementation can be detrimental in infections by intracellular pathogens including influenza A and herpes simplex viruses by suppressing immune cell responses needed to eradicate infected cells ${ }^{(24)}$, although a study of mice showed no effect on $n$-3 PUFA on responses to vaccinia virus infection ${ }^{(25)}$. Evidence on the effects of PUFA on acute febrile illnesses in humans is scant. Some studies suggest that $n$ - 3 PUFA may reduce the incidence of pneumococcal infection in the elderly and $n-3$ PUFA supplementation to infants or school-age children has resulted in decreased respiratory morbidity ${ }^{(26-31)}$, possibly of viral aetiology. We also noted that DHA was positively associated with thrombocytopenia at baseline. Whether this could be a mechanism to explain a potential effect on progression to severe disease is a matter of speculation. DHA might dampen the procoagulant function of platelets through altered synthesis of thrombin precursor proteins $^{(32)}$; nevertheless, there is no evidence to suggest that it may affect platelet numbers. Additional in vitro and nonhuman experimentation is needed to clarify the role of $n-3$ PUFA in DENV infection before considering a potential therapeutic role for these nutrients.

The inverse associations of pentadecanoic acid and DGLA with progression to DHF/DSS are novel findings. Pentadecanoic acid is an odd-chain SFA that, together with heptadecanoic acid (C17:0), represents dairy fat intake ${ }^{(33)}$; its endogenous production is negligible ${ }^{(34)}$. Epidemiological studies have consistently shown inverse associations of pentadecanoic and heptadecanoic acid biomarkers with long-term cardiometabolic disorders ${ }^{(35)}$, but the biological mechanisms underlying these relations are uncertain. Investigators have posited that one of the biological functions of pentadecanoic acid involves the regulation of circulating propionic acid $^{(34)}$, a short-chain SFA produced by intestinal bacteria through fermentation of dietary fibre. Propionic acid and other short-chain SFA may be potential mediators of the effects of microbiota on intestinal immunity and inflammation. These FA regulate leucocytes' recruitment, migration and activation through production of cytokines, chemokines and eicosanoids ${ }^{(36)}$. Whether these effects extend systemically in the event of an acute infection such as dengue remains to be elucidated. We observed a decrease in palmitic acid, the most abundant SFA, with a concomitant increase in palmitoleic acid from the acute episode to post-convalescence. Palmitic acid can be synthesised endogenously or ingested from diet, whereas palmitoleic acid can be formed from palmitic acid by the SCD. If diet remained constant, one could speculate that SCD activity might be dampened during the acute dengue episode, but the clinical implications of this potential effect are uncertain.

DGLA is a long-chain $n$-6 PUFA synthesised endogenously from linoleic acid through desaturation and elongation. D5D can convert DGLA into AA, a pro-inflammatory FA and other acute viral infections, including influenza, induce up-regulation of the lipoxygenase pathway increasing the synthesis of proinflammatory oxylipins derived from $\mathrm{AA}^{(37)}$. Nevertheless, DGLA is generally considered anti-inflammatory because it can interfere with eicosanoid biosynthesis and can be converted to prostaglandin $\mathrm{E}_{1}$, a suppressor of chronic inflammation ${ }^{(13)}$. An increase in DGLA relative to AA could acutely attenuate the synthesis of pro-inflammatory eicosanoids derived from AA, including four-series leukotrienes, two-series prostaglandins and platelet-activating factor, which may be involved in the pathophysiology of severe dengue ${ }^{(7)}$. Consistent with this possibility, increased activity of D5D, the enzyme that converts DGLA into AA, was related to higher odds of progression to DHF/DSS. We found that DGLA was inversely correlated with IL-10 at baseline, especially among cases, and elevated IL-10 seemed to predict progression to DHF/DSS in this and other studies ${ }^{(38,39)}$. This could 
indicate that a potential protective effect of DGLA on progression to severe dengue might be mediated through reduced inflammation. Another intriguing potential mechanism could be related to virucidal activity against encapsulated viruses that some longchain $n-6$ PUFA have shown in vitro ${ }^{(40-43)}$. DGLA breastmilk concentrations were inversely related to cell-free and cellassociated HIV load in milk in a study of Tanzanian HIVinfected women ${ }^{(44)}$. Non-causal explanations are also plausible. We noted that DGLA concentrations were lower during the acute episode than in an apparently disease-free period in a subgroup of participants. Assuming there were no major changes in diet between the two measurements, this opens the possibility that acute inflammation during the early stages of the disease influences DGLA concentrations and that an inverse association of DGLA with progression to severe dengue be only a reflection of early inflammatory status. Notwithstanding this possibility, DGLA supplementation increases blood DGLA in a dose-response manner ${ }^{(45,46)}$ and should not be discarded as a potential intervention that could be considered for testing in the treatment of acute DF.

This study has several strengths. Reverse causation bias was minimised by the prospective nature of the design. Recall bias was avoided with the use of objective measures of exposure. We had an opportunity to control for important potential confounders, including inflammatory biomarkers that predict progression of the disease to severe forms. We compared exposure status between the acute episode and an apparently healthy period in a subsample of participants; this approach is stronger than using a different set of healthy controls. Some limitations are also worth noting. Selection bias could occur if the selection of controls is not independent of exposure status. We noted that selected controls differed from the cohort's non-cases in some characteristics that could be related to FA status, including body weight; thus, it is not possible to completely rule out selection bias. Second, confounding could have occurred if an unmeasured predisposing factor for progression was related to FA concentrations at the onset of the febrile episode. Third, because FA concentrations are conventionally expressed as percent of total FA in the sample, associations observed with higher levels of a given FA could also represent those due to lower levels of another and vice versa. Fourth, pentadecanoic acid is present at low concentrations which are prone to measurement error. The confidence bounds around the OR were wide. Nevertheless, the mean serum C15:0 concentration in this population, $0.16 \%$, was the same or very close as that reported in other settings including Costa Rica ${ }^{(47)}$ and the United States ${ }^{(48-50)}$, lending support to the external validity of this finding. Fifth, random error may have occurred due to differences in the timing of the last meal since the acute serum sample in this study was not always a fasting sample. Serum FA concentrations do not necessarily reflect long-term intake as some may change from day to day depending on the FA composition of recent meals. Correlations between long-term intake and serum concentrations vary for different FA. They are moderate to high for exogenous FA including odd chain SFA and essential PUFA (ALA and LA) or for endogenous FA that may nevertheless be abundant in diet such as oleic acid, EPA and $\mathrm{DHA}^{(47)}$. By contrast, serum FA that are not common in the diet and mostly reflect endogenous hepatic metabolism, such as GLA and DGLA, may not represent long-term intake ${ }^{(47)}$. Sixth, the subsample of patients with postconvalescence samples was not comparable with the rest of participants with regard to some characteristics. Finally, lack of sample volume and funding constraints prevented us from determining serotype in all study subjects. DENV serotype is a strong predictor of progression to severe disease and could have confounded the associations if it were also related to exposure status. Nonetheless, the data do not support this notion because only DHA was related to both progression and serotype in the subset with available information, and the directions of the serotype-DHA and serotypeDHF/DSS associations would have caused an attenuation rather than an exaggeration of a positive DHA-DHF/DSS relation.

In sum, serum DHA concentrations at the early stages of DF are positively associated with progression to DHF/DSS whereas pentadecanoic acid and DGLA concentrations are inversely related to progression. Serum FA concentrations differ between an acute dengue episode and an apparently healthy period. These results should be confirmed in other populations as the next step in identifying FA as eventual therapeutic targets in patients with DF.

\section{Acknowledgements}

This study was supported by the National Institute of Allergy and Infectious Diseases (R21AI103364) and the Colombian Administrative Department of Science, Technology and Innovation - Colciencias (1102-04-12919, 1102-04-18205, 1102-45921561 and BPIN 2013000100011). The funding source had no role in the design, analysis or writing of this article.

E. V. and L. A. V. designed the research. L. A. V., A. L.-P. and O. F. H. conducted the research. V. M. H. conducted data management and quality assurance. E. V. performed the statistical analyses, wrote the manuscript and had primary responsibility for the final content. All authors have read and approved the final version of the manuscript.

The authors declare that there are no conflicts of interest.

\section{Supplementary material}

For supplementary material/s referred to in this article, please visit https://doi.org/10.1017/S0007114518002039

\section{References}

1. L'Azou M, Moureau A, Sarti E, et al. (2016) Symptomatic dengue in children in 10 Asian and Latin American countries. $N$ Engl J Med 374, 1155-1166.

2. Wilson ME \& Chen LH (2015) Dengue: update on epidemiology. Curr Infect Dis Rep 17, 457.

3. Guzman MG, Alvarez M \& Halstead SB (2013) Secondary infection as a risk factor for dengue hemorrhagic fever/dengue shock syndrome: an historical perspective and role of antibody-dependent enhancement of infection. Arch Virol 158, 1445-1459.

4. Vaish A, Verma S, Agarwal A, et al. (2012) Effect of vitamin E on thrombocytopenia in dengue fever. Ann Trop Med Public Health 5, 282-285. 
5. Villamor E, Villar LA, Lozano A, et al. (2017) Vitamin D serostatus and dengue fever progression to dengue hemorrhagic fever/dengue shock syndrome. Epidemiol Infect $\mathbf{1 4 5}$ 2961-2970.

6. Alagarasu K, Bachal RV, Bhagat AB, et al. (2012) Elevated levels of vitamin $\mathrm{D}$ and deficiency of mannose binding lectin in dengue hemorrhagic fever. Virol J 9, 86.

7. Voge NV, Perera R, Mahapatra S, et al. (2016) Metabolomicsbased discovery of small molecule biomarkers in serum associated with dengue virus infections and disease outcomes. PLoS Negl Trop Dis 10, e0004449.

8. Yuliana N, Fadil RMR \& Chairulfatah A (2009) Serum zinc levels and clinical severity of dengue infection in children. Paediatr Indones 49, 309-314.

9. Soundravally R, Sherin J, Agieshkumar BP, et al. (2015) Serum levels of copper and iron in dengue fever. Rev Inst Med Trop Sao Paulo 57, 315-320.

10. Turk HF \& Chapkin RS (2013) Membrane lipid raft organization is uniquely modified by $n-3$ polyunsaturated fatty acids. Prostaglandins Leukot Essent Fatty Acids 88, 43-47.

11. Garcia Cordero J, Leon Juarez M, Gonzalez YMJA, et al. (2014) Caveolin-1 in lipid rafts interacts with dengue virus NS3 during polyprotein processing and replication in HMEC-1 cells. PLOS ONE 9, e90704.

12. Calder PC (2013) Omega-3 polyunsaturated fatty acids and inflammatory processes: nutrition or pharmacology? Br J Clin Pharmacol 75, 645-662.

13. Wang $\mathrm{X}$, Lin H \& Gu Y (2012) Multiple roles of dihomogamma-linolenic acid against proliferation diseases. Lipids Health Dis 11, 25.

14. Cui L, Lee YH, Kumar Y, et al. (2013) Serum metabolome and lipidome changes in adult patients with primary dengue infection. PLoS Negl Trop Dis 7, e2373.

15. Khedr A, Hegazy M, Kamal A, et al. (2015) Profiling of esterified fatty acids as biomarkers in the blood of dengue fever patients using a microliter-scale extraction followed by gas chromatography and mass spectrometry. J Sep Sci 38, 316-324.

16. World Health Organization (1997) Dengue Haemorrhagic Fever: Diagnosis, Treatment, Prevention and Control, 2nd ed. Geneva: WHO

17. Rigau-Perez JG \& Bonilla GL (1999) An evaluation of modified case definitions for the detection of dengue hemorrhagic fever. Puerto Rico Association of Epidemiologists. $P R$ Health Sci J 18, 347-352.

18. Diaz-Quijano FA, Martinez-Vega RA \& Villar-Centeno LA (2005) Early indicators of severity in dengue virus infection. Enferm Infecc Microbiol Clin 23, 529-532.

19. Bligh EG \& Dyer WJ (1959) A rapid method of total lipid extraction and purification. Can J Biochem Physiol 37, 911917.

20. Morrison WR \& Smith LM (1964) Preparation of fatty acid methyl esters and dimethyl acetals from lipids with boron trifluoride-methanol. J Lipid Res 5, 600-608.

21. Lanciotti RS, Calisher CH, Gubler DJ, et al. (1992) Rapid detection and typing of dengue viruses from clinical samples by using reverse transcriptase-polymerase chain reaction. J Clin Microbiol 30, 545-551.

22. Santiago GA, Vergne E, Quiles Y, et al. (2013) Analytical and clinical performance of the CDC real time RT-PCR assay for detection and typing of dengue virus. PLoS Negl Trop Dis 7, e2311.

23. Vaughn DW, Green S, Kalayanarooj S, et al. (2000) Dengue viremia titer, antibody response pattern, and virus serotype correlate with disease severity. J Infect Dis 181, 2-9.
24. Husson MO, Ley D, Portal C, et al. (2016) Modulation of host defence against bacterial and viral infections by omega-3 polyunsaturated fatty acids. $J$ Infect $\mathbf{7 3}, 523-535$.

25. Jones GJB \& Roper RL (2017) The effects of diets enriched in omega-3 polyunsaturated fatty acids on systemic vaccinia virus infection. Sci Rep 7, 15999.

26. Birch EE, Hoffman DR, Castaneda YS, et al. (2002) A randomized controlled trial of long-chain polyunsaturated fatty acid supplementation of formula in term infants after weaning at 6 wk of age. Am J Clin Nutr 75, 570-580.

27. Pastor N, Soler B, Mitmesser SH, et al. (2006) Infants fed docosahexaenoic acid- and arachidonic acid-supplemented formula have decreased incidence of bronchiolitis/bronchitis the first year of life. Clin Pediatr (Phila) 45, 850-855.

28. Thienprasert A, Samuhaseneetoo S, Popplestone K, et al. (2009) Fish oil $n-3$ polyunsaturated fatty acids selectively affect plasma cytokines and decrease illness in Thai schoolchildren: a randomized, double-blind, placebo-controlled intervention trial. J Pediatr 154, 391-395.

29. Minns LM, Kerling EH, Neely MR, et al. (2010) Toddler formula supplemented with docosahexaenoic acid (DHA) improves DHA status and respiratory health in a randomized, double-blind, controlled trial of US children less than 3 years of age. Prostaglandins Leukot Essent Fatty Acids $\mathbf{8 2}$, 287-293.

30. Malan L, Baumgartner J, Calder PC, et al. (2015) n-3 Longchain PUFAs reduce respiratory morbidity caused by iron supplementation in iron-deficient South African schoolchildren: a randomized, double-blind, placebo-controlled intervention. Am J Clin Nutr 101, 668-679.

31. Imhoff-Kunsch B, Stein AD, Martorell R, et al. (2011) Prenatal docosahexaenoic acid supplementation and infant morbidity: randomized controlled trial. Pediatrics $\mathbf{1 2 8}$, e505-e512.

32. Larson MK, Tormoen GW, Weaver LJ, et al. (2013) Exogenous modification of platelet membranes with the omega-3 fatty acids EPA and DHA reduces platelet procoagulant activity and thrombus formation. Am J Physiol Cell Physiol 304, C273-C279.

33. Wolk A, Furuheim M \& Vessby B (2001) Fatty acid composition of adipose tissue and serum lipids are valid biological markers of dairy fat intake in men. J Nutr 131, 828-833.

34. Pfeuffer M \& Jaudszus A (2016) Pentadecanoic and heptadecanoic acids: multifaceted odd-chain fatty acids. Adv Nutr $\mathbf{7}$, 730-734.

35. Riserus U \& Marklund M (2017) Milk fat biomarkers and cardiometabolic disease. Curr Opin Lipidol 28, 46-51.

36. Vinolo MA, Rodrigues HG, Nachbar RT, et al. (2011) Regulation of inflammation by short chain fatty acids. Nutrients $\mathbf{3}$, 858-876.

37. Cui L, Fang J, Ooi EE, et al. (2017) Serial metabolome changes in a prospective cohort of subjects with influenza viral infection and comparison with dengue fever. J Proteome Res 16, 2614-2622.

38. Malavige GN, Gomes L, Alles L, et al. (2013) Serum IL-10 as a marker of severe dengue infection. BMC Infect Dis 13, 341.

39. Liao B, Tang Y, Hu F, et al. (2015) Serum levels of soluble vascular cell adhesion molecules may correlate with the severity of dengue virus-1 infection in adults. Emerg Microbes Infect $\mathbf{4}$, e24.

40. Kohn A, Gitelman J \& Inbar M (1980) Unsaturated free fatty acids inactivate animal enveloped viruses. Arch Virol 66 , 301-307.

41. Horowitz B, Piet MP, Prince AM, et al. (1988) Inactivation of lipid-enveloped viruses in labile blood derivatives by unsaturated fatty acids. Vox Sang 54, 14-20. 
42. Thormar H, Isaacs CE, Kim KS, et al. (1994) Inactivation of visna virus and other enveloped viruses by free fatty acids and monoglycerides. Ann N Y Acad Sci 724, 465-471.

43. Hilmarsson H, Kristmundsdottir T \& Thormar H (2005) Virucidal activities of medium- and long-chain fatty alcohols, fatty acids and monoglycerides against herpes simplex virus types 1 and 2: comparison at different $\mathrm{pH}$ levels. APMIS 113, 58-65.

44. Villamor E, Koulinska IN, Furtado J, et al. (2007) Long-chain $n-6$ polyunsaturated fatty acids in breast milk decrease the risk of HIV transmission through breastfeeding. Am J Clin Nutr $\mathbf{8 6}$, 682-689.

45. Tanaka T, Kakutani S, Horikawa C, et al. (2012) Oral supplementation with dihomo-gamma-linolenic acid (DGLA)enriched oil increases serum DGLA content in healthy adults. Lipids 47, 643-646.

46. Teraoka N, Kawashima H, Shiraishi-Tateishi A, et al. (2009) Oral supplementation with dihomo-gamma-linolenic acidenriched oil altered serum fatty acids in healthy men. Biosci Biotechnol Biochem 73, 1453-1455.
47. Baylin A, Kim MK, Donovan-Palmer A, et al. (2005) Fasting whole blood as a biomarker of essential fatty acid intake in epidemiologic studies: comparison with adipose tissue and plasma. Am J Epidemiol 162, 373-381.

48. de Oliveira Otto MC, Nettleton JA, Lemaitre RN, et al. (2013) Biomarkers of dairy fatty acids and risk of cardiovascular disease in the multi-ethnic study of atherosclerosis. J Am Heart Assoc 2, e000092.

49. Yaemsiri S, Sen S, Tinker LF, et al. (2013) Serum fatty acids and incidence of ischemic stroke among postmenopausal women. Stroke 44, 2710-2717.

50. Yakoob MY, Shi P, Willett WC, et al. (2016) Circulating biomarkers of dairy fat and risk of incident diabetes mellitus among men and women in the United States in two large prospective cohorts. Circulation 133, 1645-1654.

51. de Onis M, Onyango AW, Borghi E, et al. (2007) Development of a WHO growth reference for school-aged children and adolescents. Bull World Health Organ 85, 660-667. 